\author{
J. GRAF ${ }^{1, \infty}$ \\ B.S. LUK'YANCHUK ${ }^{2}$ \\ M. MOSBACHER ${ }^{1}$ \\ M.H. $\mathrm{HONG}^{2}$ \\ C.T. $\mathrm{CHONG}^{2}$ \\ J. BONEBERG ${ }^{1}$ \\ P. LEIDERER $^{1}$
}

\section{Matrix laser cleaning: a new technique for the removal of nanometer sized particles from semiconductors}

\author{
${ }^{1}$ University of Konstanz, Universitaetsstrasse 10, 78457 Konstanz, Germany \\ ${ }^{2}$ Data Storage Institute, Agency for Science, Technology and Research, 117608 Singapore
}

Received: 22 March 2007/Accepted: 23 March 2007

Published online: 17 May 2007 • @ Springer-Verlag 2007

ABSTRACT In this paper a new laser-based technique for the removal of nanoparticles from silicon wafers, called matrix laser cleaning, is introduced. In contrast to the already existing technique dry laser cleaning damages of the substrate can be avoided. Furthermore no liquids are used, avoiding problems that occur, e.g. in steam laser cleaning and other wet cleaning techniques. We show that damage free particle removal of polystyrene particles with diameters of at least down to $50 \mathrm{~nm}$ is possible with a cleaning efficiency very close to $100 \%$ within a single shot experiment. Furthermore the cleaning threshold is independent of the particle size.

PACS 64.70.Hz; 68.43.Vx; 81.65.Cf
1

\section{Introduction}

The removal of nanoparticles from semiconductor and other delicate surfaces is of great interest for applications in the semiconductor industry and nanotechnology. The ongoing trend to further miniaturisation decreases the size of particles that can produce defects in electronic circuits or nanostructures furthermore. According to the international roadmap for semiconductors [1] in 2009 a cleaning technique should be able to remove particles down to at least $35-50 \mathrm{~nm}$ in diameter. Despite the progress in the conventional techniques like megasonics $[2,3]$ there are still future cleaning problems that have not yet been solved. One alternative cleaning approach that might solve such future problems is called laser cleaning [4], where the sample to be cleaned is irradiated with a short, in most cases nanosecond, laser pulse. The advantages of this technique are cost efficiency, environmental friendliness and the fact that this is a contact free cleaning method where single areas of a wafer or a lithographic mask can be selectively addressed. So far two major laser cleaning approaches can be distinguished. The first one is called dry laser cleaning (DLC), where the surface to be cleaned is directly illuminated by a laser pulse without any pre-treatment or additional layer [5]. At first it was assumed that the acceleration forces produced by thermal expansion of the substrate and the particle due to absorption of the applied irradiation are sufficient to remove the particles. However, detailed analysis shows small defects at the former particle positions [6-8]. These defects are created by local ablation underneath the particles due to a near field enhancement and constitutes an additional cleaning mechanism [9]. Furthermore it has been shown that this mechanism is the dominant one [10], so that to our knowledge for all laser parameters investigated so far within DLC no damage free particle removal has been achieved. In the second approach, called steam laser cleaning (SLC), a liquid, in most cases water or water/alcohol mixture, is applied to the sample prior to the laser pulse $[11,12]$. The laser energy gets either directly absorbed inside the liquid or transferred from the substrate via heat transfer, leading to bubble nucleation and a subsequent explosive evaporation of the liquid layer. In this way we achieved particle removal of polystyrene particles with diameters down to $140 \mathrm{~nm}$, without damaging the substrate [13]. Nevertheless liquids are not wanted in the semiconductor industry due to various reasons, e.g. the risk of watermark formation or possible damage of structures due to capillary forces. Furthermore a complete wetting of the substrate is an inevitable prerequisite and complex drying steps have to be added after the cleaning process. In this paper we introduce a third and new technique, called matrix laser cleaning that incorporates all the advantages of SLC without the use of liquids.

2

\section{Basic idea}

The principal idea is to embed the particles into a matrix of a gas condensed at low temperatures onto the sample into the solid phase (see Fig. 1a). Thereby, the used gas must fulfil the following specifications:

1. At first the pressure of the triple point should lie above the working conditions. Thus, the matrix sublimates instead of going through the liquid phase, so that the problems mentioned in Sect. 1 can be avoided. In fact, depending on the substrate, dewetting can also occur for the quench condensed molecular solids that are used in our technique. Nevertheless these wetting transitions are on the timescale of minutes and 




FIGURE 1 Sketch of the experimental situation. Before the laser pulse (a) the particles were embedded in a solid matrix. After the laser shot (b) a small part of the matrix in the vicinity to the substrate undergoes a phase transition forming an expanding vapour layer acting on the particles. In addition the major part of the matrix leaves the surface as an intact layer, resulting in a drag force acting on the particles due to adhesion forces

above and therefore far beyond the timescale of our cleaning process.

2. Second the solid layer should be transparent for the applied laser wavelength. Thus the laser energy gets absorbed inside the sample and solely coupled into the matrix film by heat transfer. This leads to a strongly inhomogeneous temperature profile with its maximum at the interface to the sample and a decay on the order of the thermal diffusion length $l_{\tau} \approx 2 \sqrt{D \tau}$ (with the heat diffusivity $D$ and the laser pulse length $\tau)$. As long as the film thickness $d$ is larger than $l_{\tau}$ (this is the case in all our experiments) only a small fraction of the film in the vicinity of the substrate undergoes a phase transition, producing a compressed vapour layer with initial thickness $d_{\text {vap }}$ acting on the particles as well as the adjacent solid layer (see Fig. 1b).

We could show by interferometric measurements that the solid part of the matrix leaves the surface as an in- tact layer, as in the case of a liquid film $[14,15]$. This will also be the subject of future publications.

Two different cleaning mechanisms contribute to particle removal.

The first results from the momentum transfer from the gas molecules to the particles. This mechanism also occurs if the particle diameter $2 r$ is smaller than the initial gas layer thickness $d_{\text {vap }}$, where we have a situation similar to MALDI [16]. A more detailed analysis using Euler's equation for the gas layer results in a driving force $F=2 m v r v_{0} / l_{\tau}$ with the mass $m$ of a gas molecule, the collision frequency $v$, the particle radius $r$, the starting velocity $v_{0}$ of the solid layer and the thermal diffusion length $l_{\tau}$. The second cleaning mechanism only occurs if the particle diameter $2 r$ is larger than the initial gas layer thickness $d_{\text {vap }}$. Since $d_{\text {vap }}$ is assumed to be on the order of a few namometers as in the case of a liquid film [15], this condition is fulfilled for all technically relevant particle sizes. Thus the upper part of the particles stays embedded inside the still solid part of the matrix leaving the surface, resulting in a drag force based on van der Waals forces. Since the contact area between the matrix and the particle exceeds the one between the particle and the substrate by far, this drag force should also exceed the adhesion forces between particle and substrate.

\section{$3 \quad$ Experimental realization and results}

In the following we present our experimental setup, as well as the first cleaning results for polystyrene particles. A frequency doubled $\mathrm{Nd}$ :YAG laser (532 nm, 9 ns FWHM) was used as cleaning laser. As matrix material we chose carbon dioxide. To prevent condensation of unwanted species all experiments were carried out in a custom built vacuum chamber. Furthermore the chamber was flooded with carbon dioxide several times before each measurement. The solid films were prepared by a quench condensation technique. Therefore, the samples were mounted on a sample holder cooled by liquid nitrogen down to temperatures between 135 and $175 \mathrm{~K}$. After the desired temperature was reached a gaseous flow of carbon dioxide was blown over the sample leading to condensation of the solid film with condensation rates between 40 and $350 \mathrm{ML} / \mathrm{s}$. The films were controlled in situ by optical techniques. From the fact that no scattered light was detected during the whole film condensation process we conclude that the films are homogenous on the order of optical wavelengths and thus effective medium theories can be applied. Furthermore, reflectometry allows to control the film thickness with an accuracy of $3 \mathrm{~nm}$. In addition, the amplitudes in the reflectometry measurements yield an optical refractive index for the matrix of $n=1.31$ at $660 \mathrm{~nm}$. In combination with the value of $n=1.41$ for solid $\mathrm{CO}_{2}$ [17] this leads to solid fractions inside the matrix of $77 \%$ according to Bruggemann [18] and 78\% according to the Maxwell-Garnett theory [19]. It has been shown by Falk [20] and Weida [21] that $\mathrm{CO}_{2}$ films condensed at growth rates between 36 and $105 \mathrm{ML} / \mathrm{s}$ and substrate temperatures $T>50 \mathrm{~K}$ (confirmed experimentally up to $120 \mathrm{~K}$ ) exhibit a polycrystalline structure. Since our films were prepared at the same growth rates and only slightly higher temperatures we presume that these possess a polycrystalline structure with a porosity around $20 \%$.

If one measures the laser fluences necessary for particle removal over the applied $\mathrm{CO}_{2}$ film thickness an oscillatory behaviour with a periodicity of about $185 \mathrm{~nm}$ is found. This behaviour becomes clear if one considers condition (2) in Sect. 2. The laser energy is only absorbed inside the substrate and the solid matrix just acts as a transparent overlayer. Due to an interference effect this overlayer leads to a thicknessdependent reflectivity $R(d)$ and thus also a thickness-dependent transmissivity into the substrate. Therefore, the applied laser fluences have to be normalized by the varying transmissivity to receive the fluence absorbed inside the substrate, denoted as "effective laser fluence" $F_{\text {eff }}=F(1-R(d))$ in the following.

Considering the effective cleaning fluences for rather large particles with diameters above $300 \mathrm{~nm}$ reveals that an applied layer of $\mathrm{CO}_{2}$ does not contribute to particle removal at all. The effective fluence for particle removal for all investigated film thicknesses equals the one with zero film thickness. Furthermore particles with diameters above $300 \mathrm{~nm}$ 

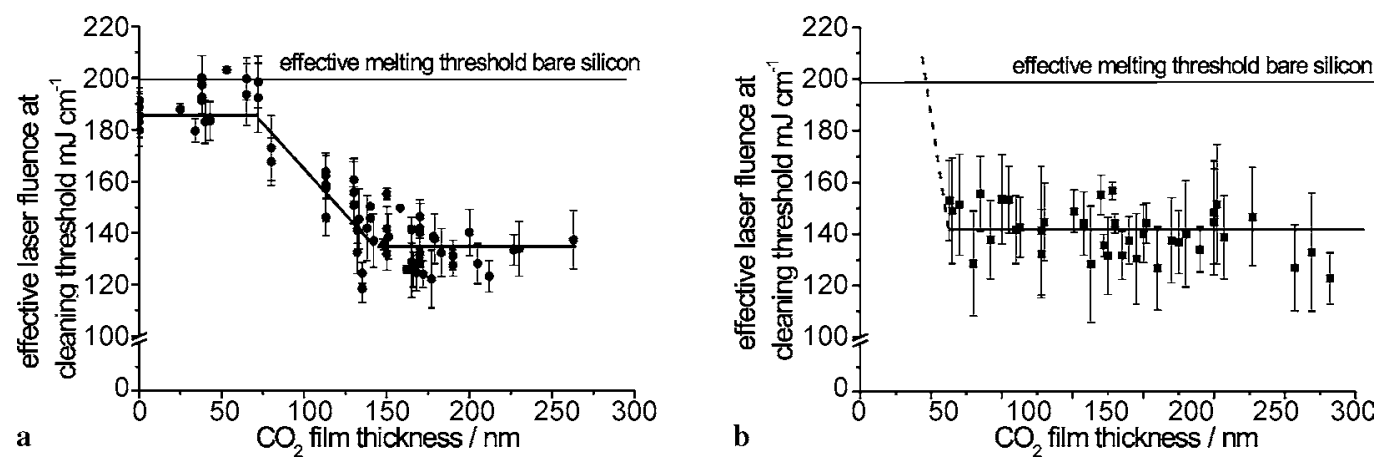

FIGURE 2 Effective laser fluence necessary for particle removal for small polystyrene particles (left: $190 \mathrm{~nm}$ in diameter, right: $50 \mathrm{~nm}$ in diameter) dependent on the applied $\mathrm{CO}_{2}$ matrix thickness $d$. Above a certain film thickness the MLC cleaning mechanism sets in. In the case of the 190 nm sized particles this results in a decrease of the cleaning threshold fluence to the universal MLC threshold. In the $50 \mathrm{~nm}$ case cleaning only occurs above this film thickness, since these particles cannot be removed by DLC

could be removed at laser fluences, where the $\mathrm{CO}_{2}$-layer still remained on the surface. Thus we conclude that large particles are removed by dry laser cleaning mechanisms.

A different picture arises for the removal of smaller particles with diameters below $300 \mathrm{~nm}$. For applied film thicknesses on the order of the respective particle radius the effective fluence for particle removal drops to a constant particle size-independent value significantly below the one for zero film thickness (Fig. 2a). Comparisons with measurements on bare $\mathrm{CO}_{2}$ matrix films show that this value equals the ablation threshold of the matrix itself, indicating the onset of an additional cleaning effect induced by the ablation of the $\mathrm{CO}_{2}$ matrix. This effect becomes even more pronounced for particles smaller than $150 \mathrm{~nm}$ in diam-

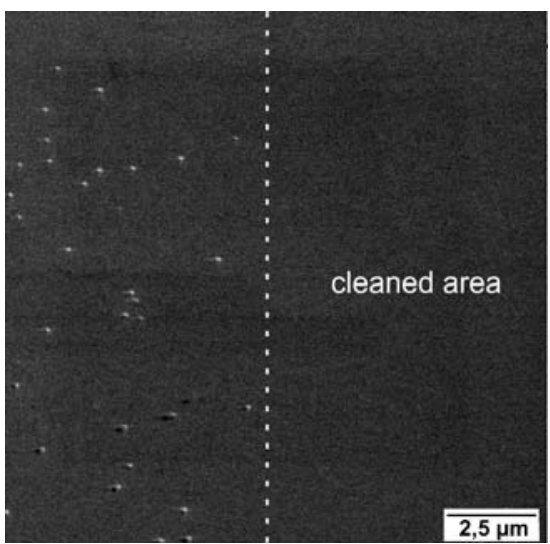

FIGURE 3 Scanning electron microscope image of the edge of an area cleaned by matrix assisted laser cleaning. On the right the particles $(50 \mathrm{~nm}$ diameter, polystyrene) have been removed with a cleaning efficiency close to $100 \%$ within a single shot experiment. Even after detailed analysis no damages to the substrate have been observed eter (Fig. 2b). These particles are no longer removable by dry laser cleaning. Nevertheless, Fig. 3 shows a scanning electron microscope image of the border of an area cleaned by matrix laser cleaning. The particle size is $50 \mathrm{~nm}$, to our knowledge the smallest particles that have ever been cleaned by laser based techniques. We achieved a cleaning efficiency very close to $100 \%$ within a single shot experiment, which leads to the assumption that the minimum size limit of particles that can be removed has not yet been reached.

\section{Discussion}

As shown above matrix laser cleaning is a cleaning method that starts working for particles smaller than $300 \mathrm{~nm}$, while for larger particles no additional cleaning effect occurs. This behaviour is quite unusual, since for smaller particles a bigger fraction contributes to the short ranged adhesion forces to the substrate and thus particles normally become harder to remove the smaller they are. A reason for this can be found in a comparison between the $\mathrm{CO}_{2}$ ablation threshold (and thus also the threshold for matrix laser cleaning) and the dry laser cleaning thresholds for different particle sizes. As can bee seen in Fig. 4 for large particle sizes the DLC threshold lies far below the ablation threshold of the $\mathrm{CO}_{2}$ matrix. Therefore the particles are removed by the DLC mechanism even at fluences where the matrix stays on the surface. Indeed, in these cases damages similar to those found in DLC [22] are found at the former particle positions. However, due to the increasing DLC threshold for decreasing particle size [23] at a particle size around $300 \mathrm{~nm}$ the DLC threshold fluence intersects the $\mathrm{CO}_{2}$ matrix ablation threshold, leading to an additional cleaning mechanism induced by the ablating matrix. Furthermore this mechanism is supposed to be the dominant one, since even after detailed analysis no defects typical for the DLC mechanism have been found in this particle size regime.

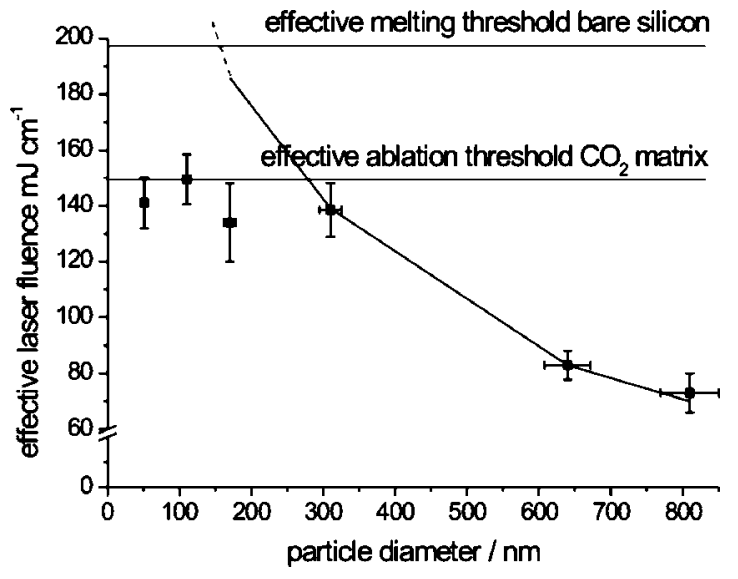

FIGURE 4 Comparison of the effective cleaning threshold for matrix laser cleaning and dry laser cleaning at the same substrate temperature for different particle sizes. For larger particle diameters the DLC threshold (solid line) lies far below the $\mathrm{CO}_{2}$ ablation threshold. For decreasing particle size the DLC threshold increases, leading to an intersection with the $\mathrm{CO}_{2}$ ablation threshold around particle sizes of $300 \mathrm{~nm}$. Thus matrix laser cleaning starts to work at particle sizes below $300 \mathrm{~nm}$ 
We are aware of the fact that the choice of carbon dioxide as matrix material might not be the optimal one with respect to applications. At first the samples have to be cooled down to about $170 \mathrm{~K}$ before quench condensation of the matrix becomes possible. Second, the ablation threshold and thus also the cleaning threshold is quite close to the melting threshold of bare silicon, bearing the danger of substrate damage. Reasons for this might be found in a bad thermal contact with the underlying silicon sample or the dynamics of the induced phase transition itself, which will be the subject of further investigations. Therefore, with respect to industrial applications there might be more suitable matrix materials than carbon dioxide with a lower ablation threshold and a higher condensation temperature (among the possible candidates might be materials like naphthalene). Also, the possibility to remove particles from substrates other than silicon and even structured substrates will be the objectives of future experiments. Nevertheless, we already show that matrix laser cleaning is a powerful tool for damage free removal of the smallest particles from silicon wafers (see also some preliminary results in [24]). In combination with a conventional pre- cleaning step followed by a selective matrix laser cleaning step of the remaining particles, this technique might be an alternative to conventional techniques in the future.

ACKNOWLEDGEMENTS The authors thank Florian Lang for valuable discussions. Financial support by the Center for Applied Photonics (CAP) at the University of Konstanz and the Deutsche Forschungsgemeinschaft (SFB 513) is gratefully acknowledged.

\section{REFERENCES}

1 The International Technology Roadmap for Semiconductors, http://public.itrs.net. Cited 2007

2 G. Vereecke, F. Holsteyns, S. Arnauts, S. Beckx, P. Jaenen, K. Kenis, M. Lismont, M. Lux, R. Vos, J. Snow, P. Mertens, Solid State Phenom. 103-104, 141 (2005)

3 F. Holsteyns, K. Lee, S. Graf, R. Palmans, G. Vereecke, P. Mertens, Solid State Phenom. 103-104, 159 (2005)

4 B.S. Luk'yanchuk, Laser Cleaning (World Scientific, London, 2002)

5 A.C. Engelsberg, Dissertation, Rensselaer Polytechnic Institute (1988)

6 P. Leiderer, J. Boneberg, V. Dobler, M. Mosbacher, H.-J. Muenzer, N. Chaoui, J. Siegel, C.N. Afonso, T. Fourier, G. Schrems, D. Baeuerle, Proc. SPIE 4065, 249 (2000)

7 M. Mosbacher, H.-J. Muenzer, J. Zimmermann, J. Solis, J. Boneberg, P. Leiderer, Appl. Phys. A 72, 41 (2001)

8 S.M. Huang, M.H. Hong, Y.F. Lu, B.S. Luk'yanchuk, W.D. Song, T.C. Chong, J. Appl. Phys. 92, 2495 (2002)
9 M. Mosbacher, M. Bertsch, H.-J. Muenzer, V. Dobler, B.-U. Runge, D. Baeuerle, J. Boneberg, P. Leiderer, Proc. SPIE 4426, $308(2002)$

10 J. Graf, F. Lang, M. Mosbacher, P. Leiderer, Solid State Phenom. 103-104, 185 (2005)

11 K. Imen, S.J. Lee, S.D. Allen, Appl. Phys. Lett. 58, 203 (1991)

12 W. Zapka, W. Ziemlich, A.C. Tam, Appl. Phys. Lett. 58, 2217 (1991)

13 F. Lang, M. Mosbacher, P. Leiderer, Appl. Phys. A 77, 117 (2003)

14 K.M. Smith, M.Y. Hussaini, L.D. Gelb, S.D. Allen, Appl. Phys. A 77, 877 (2003)

15 F. Lang, P. Leiderer, New J. Phys. 8, 14 (2006)

16 A.A. Puretzky, D.B. Geohegan, G.B. Hurst, M.V. Buchanan, B.S. Luk'yanchuk, Phys. Rev. Lett. 83, 444 (1999)

17 S.G. Warren, Appl. Opt. 25, 2650 (1986)

18 D.A.G. Bruggeman, Ann. Phys. 24, 636 (1935)

19 J.C.M. Garnett, Philos. Trans. R. Soc. London 203, 385 (1904)

20 M. Falk, J. Chem. Phys. 86, 560 (1987)

21 M.J. Weida, J.M. Sperhac, D.J. Nesbitt, J. Chem. Phys. 105, 749 (1996)

22 H.-J. Münzer, M. Mosbacher, M. Bertsch, J. Zimmermann, P. Leiderer, J. Boneberg, J. Microsc. 202, 129 (2001)

23 M. Mosbacher, V. Dobler, M. Bertsch, H.-J. Münzer, J. Boneberg, P. Leiderer, in Surface Contamination and Cleaning, ed. by K.L. Mittal (VSP, Utrecht, Boston, 2003) pp. 311-334

24 B.S. Luk'yanchuk, W.D. Song, Z.B. Wang, Y. Zhou, M.H. Hong, T.C. Chong, J. Graf, M. Mosbacher, P. Leiderer, Laser Ablation and its Applications, ed. by C. Phipps (Springer, Berlin Heidelberg New York, 2006) pp. 37-67 